Fifth International Conference on Space Structures, 19-21 August 2002, University of Surrey

\title{
Expandable Structures formed by Hinged Plates
}

\author{
F JENSEN and S PELLEGRINO, Department of Engineering, University of Cambridge, UK
}

\begin{abstract}
This paper presents a family of two-dimensional expandable structures formed by flat plates connected by cylindrical (scissor) joints. These structures are kinematically equivalent to previously known expandable bar structures. Special shapes of the plates are determined for which the plates do not overlap or interfere during the expansion of the structure, and for which the structure forms a gap-free disk in the closed configuration and an annulus in the open configuration.
\end{abstract}

\section{INTRODUCTION}

This paper is concerned with the geometric design of symmetric expandable structures consisting of rigid, flat plates connected by joints that allow only a relative rotation about one axis, i.e. cylindrical hinges or scissor joints. We are interested in assemblies of identical plates forming a complete ring, and which are able to move relative to one another between two extreme configurations. In the closed configuration these structures form a gap free disk and in the open configuration they form an annulus with, for example, a circular opening in the middle. These structures are visually pleasing and have potential applications in the design of retractable roofs.

A limited number of structures with properties broadly similar to those studied in this paper have been known for some time. Verheyen [1] made extensive studies of transformable structures based on pairs of overlapping elements connected by a cylindrical hinge in the middle, with neighbouring pairs connected by spherical joints. Wohlhart [2] and You [3] have considered expandable structures formed by rigid elements connected through hinged links. Due to the relative rotation between the elements in each pair in the former case, and between the main elements and the links in the latter case, these structures can vary in size while maintaining essentially the same geometric shape.

The approach that is presented in this paper starts from a two-dimensional expandable bar structure and replaces the bars with flat plates which are connected with scissor joints at exactly the same locations as the original bar structure. Thus, the kinematic behaviour of the bar structure is unchanged. Then, the shape and size of these plates are determined for which the largest motion of the plate structure is possible.

The layout of this paper is as follows. The next section briefly reviews existing solutions for expandable bar structures. A solution for $n$-fold symmetric structures consisting of multiangulated bars is of particular interest, and the following section obtains expressions for the inner and outer radii of this type of structure in the open and closed configurations. Then, the section Cover Elements for the Bar Structure determines certain special shapes of flat plates that can be attached to the bar structure without affecting its range of motion. This solution is then used in the following section for the design of some practical structures. A brief discussion concludes the paper. 


\section{BACKGROUND: EXPANDABLE BAR STRUCTURES}

Expandable bar structures based on the concept of scissor hinges have been known for a long time, initially only as two-dimensional, lazy-tong-type structures that extend linearly. More recently, curved structures that expand in three dimensions have been pioneered by the Spanish engineer Pinero [4] and further developed by Escrig [5] and Zeigler [6]. A major advantage of these structures compared to other expandable structures is the relative simplicity of their joints.

A considerable advance in the design of this kind of structures was made by Hoberman, with the invention of the simple angulated element [7]. In its simplest form, shown in Figure 1, this element consists of two identical angulated bars with central kinks of equal amplitude $\alpha$ ("angulated bars,,), connected by a scissor hinge at the centre, i.e. at node $E$. This element has the special property that lines through the end nodes $A, D$ and $B, \mathrm{C}$ subtend a constant angle when the angle between the two angulated bars is changed; in other words, an angulated element subtends a constant angle when it is opened and closed.

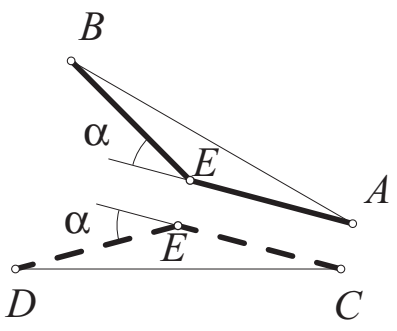

(a)

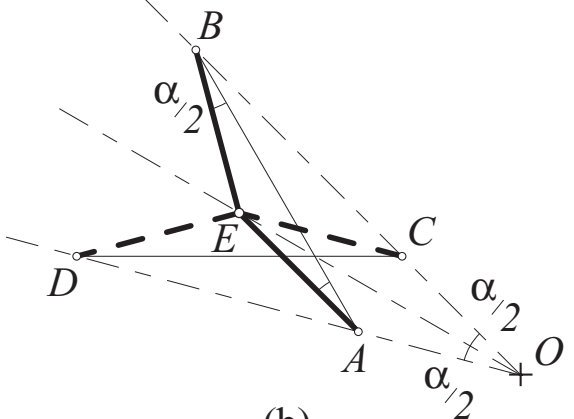

(b)

Figure 1: Angulated element

Because of this property, simple two-dimensional expandable structures can be obtained by forming one or more closed, concentric rings of identical angulated elements. For example, the expandable structure shown in Figure 2 could be made by joining with scissor hinges two such rings, each consisting of 12 elements. Three-dimensional expandable structures, such as the Hoberman sphere [8], are also formed from closed rings of identical angulated elements.
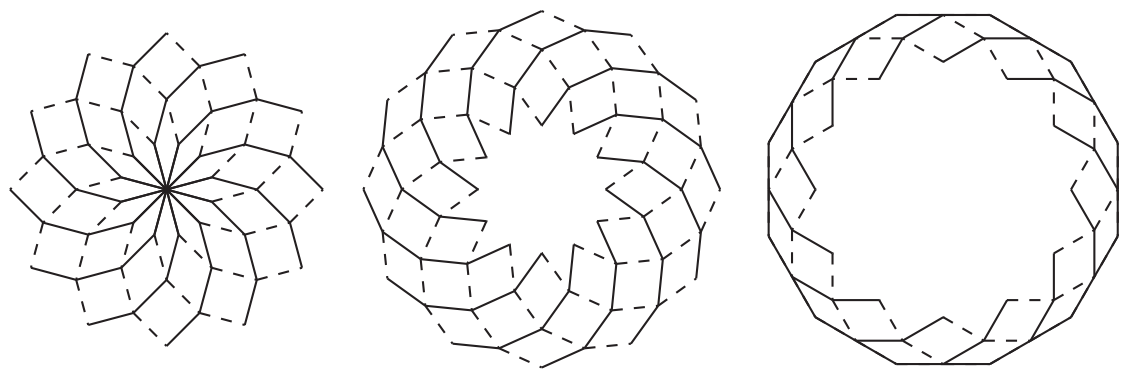

Figure 2: Expandable structure consisting of multi-angulated bars

You and Pellegrino $[9,10]$ noted that consecutive angulated bars in Figure 2 maintain a constant angle equal to $\alpha$ when the structure is expanded, and thus can be replaced with a single multi-angulated bar. Thus, the structure of Figure 2 could also be made from a total of 24 bars, each having four segments with equal kink angles: 12 bars are arranged in a clockwise direction and 12 anti-clockwise. At each cross-over point, there is a scissor joint. 
Note that the whole structure is arranged according to a pattern of identical rhombuses, which are "sheared, when the structure expands. It is also possible to design much less symmetric expandable structures, e.g. with elliptical shape [10], but this is beyond the scope of the present paper.

A general expandable structure consisting of identical multi-angulated elements, such as that shown in Figure 2, is defined by the number of segments in each angulated element, $k$, and the number of angulated elements in each layer, $n$, plus the segment length. Thus, apart from a scaling factor, this structure is fully defined by the parameters $n ; k$, see Figure 3 for an example. Note that, since the structure has $n$-fold symmetry

$$
\alpha=\frac{2 \pi}{n}
$$

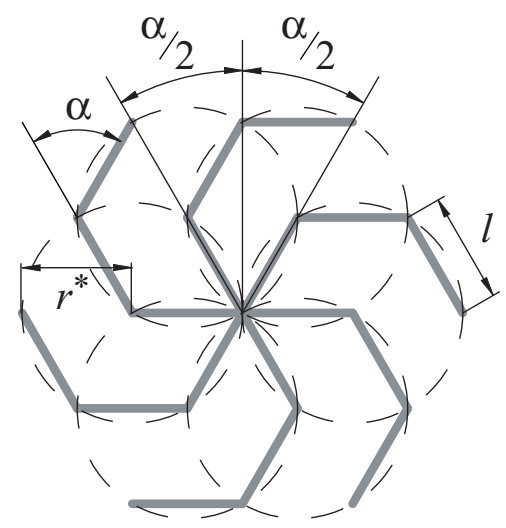

Figure 3: One layer of $6 ; 3$ structure

If the structure is allowed to rotate while it expands, i.e. the scissor hinges are not required to move on radial lines, the structure can be connected to $n$ fixed points, which are the centres of the $n$ circles of radius $r^{*}$ defined by the multi-angulated elements that make up one layer, see Figure 3. Thus, the motion of one layer of the structure is a pure rotation of each of its elements about the centre of its corresponding circle. Using $r^{*}$ to define the size of the structure, the segment length is

$$
l=2 r^{*} \sin \frac{\alpha}{2}
$$

Either rigid or flexible covering elements can be attached to the structures described above to create retractable roofs $[8,11]$, but in previous studies these elements were allowed to overlap in some, or all configurations.

\section{EXTREME CONFIGURATIONS OF BAR STRUCTURES}

The total rotation undergone by a multi-angulated element during the motion of the structure from the fully-closed to the fully-open configuration will be called the rotation angle, $\beta$. This angle can be found from Figure 4, showing the closed position. Note that all of the angulated elements meet at the centre $O$, and their circles of motion also intersect at $O$. The open configuration is reached when neighbouring multi-angulated elements touch at the point of intersection of their respective circles of motion, $P$. 


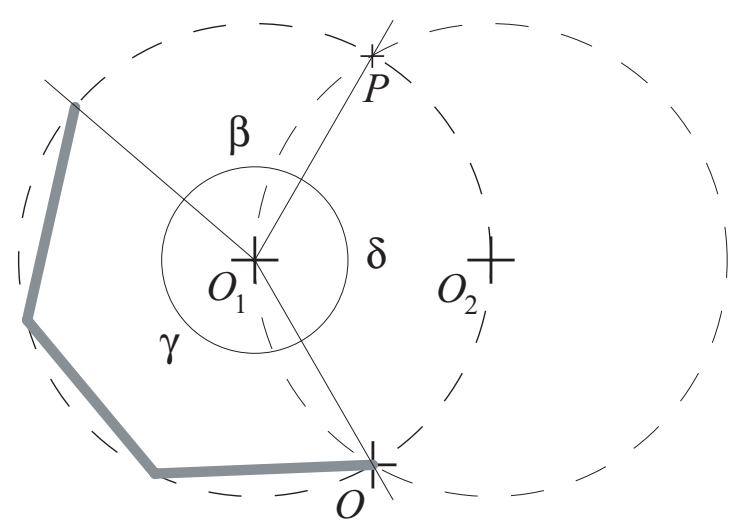

Figure 4: Definition of angles for a multi-angulated element

Because the angle subtended by a single bar on the circle of motion is equal to $\alpha$, the element angle subtended by a multi-angulated element is

$$
\gamma=k \alpha
$$

The limit angle subtended by the intersection points of two neighbouring circles of motion, $O$ and $P$, corresponds to the two limits for the motion of the multi-angulated element. It is found from:

$$
\delta=\pi-\alpha
$$

The rotation angle can then be calculated from:

$$
\beta=2 \pi-\gamma-\delta=\pi+(1-k) \alpha
$$

In practice, the bar structure will not be able to reach these extreme configurations, due to the physical size of elements and joints. Therefore, we introduce two reduction angles, $\gamma_{1}$ and $\gamma_{2}$, to denote the corrections needed respectively for the closed and open positions. The reduced rotation angle is thus

$$
\beta^{*}=\pi+(1-k) \alpha-\gamma_{1}-\gamma_{2}
$$

Now, consider the two extreme configurations of the bar structure. Denoting by $r$ and $R$ its inner and outer radii, respectively, and using the subscripts $\min$ and $\max$ for the open and closed configurations, respectively, the following expressions can be derived by analysing Figure 5:

$$
\begin{gathered}
r_{\text {max }}=2 r^{*} \sin \left[\frac{1}{2}\left(\pi+(1-k) \alpha-\gamma_{2}\right)\right]=2 r^{*} \cos \left[(k-1) \frac{\alpha}{2}+\frac{\gamma_{2}}{2}\right] \\
R_{\text {max }}=2 r^{*} \sin \left(\frac{\pi-\gamma_{2}}{2}\right)=2 r^{*} \cos \left(\frac{\gamma_{2}}{2}\right) \\
r_{\text {min }}=2 r^{*}\left(\frac{\gamma_{1}}{2}\right)
\end{gathered}
$$




$$
R_{\min }=2 r^{*}\left(\frac{v+\gamma_{1}}{2}\right)
$$

\section{Joint Size Effects}

Because the bar structure is composed of two distinct layers, the only possible interference is between elements in the same layer. Assuming the joints to be circular with radius $r_{j}$ the two reduction angles are

$$
\gamma_{1}=\gamma_{2}=2 \arcsin \left(\frac{r_{j}}{r^{*}} \frac{1}{2 \sin (\alpha / 2)}\right)
$$

\section{COVER ELEMENTS FOR BAR STRUCTURE}

We begin by considering what limitations are imposed on the motion of only a small piece of the bar structure when covering plates are attached to it.

Consider a linkage consisting of two parallel bars $A_{\mathrm{i}} A_{\mathrm{i}+1}$ and $B_{\mathrm{i}+1} B_{\mathrm{i}+2}$ and a pair of parallel linking bars, as shown in Figure 6(a). Bar $B_{\mathrm{i}+1} B_{\mathrm{i}+2}$ is assumed to be fixed so no rigid body motions are allowed and this leaves one internal mechanism which allows the linking bars to rotate and bar $A_{\mathrm{i}} A_{\mathrm{i}+1}$ to translate. The top-left angle defines the rotation angle, $\beta$, as shown in Figure 6(a); this angle is positive clockwise.

Consider a rigid plate attached to bars $A_{\mathrm{i}} A_{\mathrm{i}+1}$ and $B_{\mathrm{i}+1} B_{\mathrm{i}+2}$. This rigid body eliminates the mechanism of the parallelogram. If a straight cut at an inclination angle $\theta$ is made in the plate, then the mechanism is restored; the line of the cut is called the inclination line. So, now we have two plates attached to the linkage, which are not allowed to overlap; depending on the inclination angle, $\beta$ can either increase or decrease. In each case, the motion of the parallelogram has to stop when the gap between the two plates is closed again. The two limits on the rotation angle are denoted by $\beta_{1}$ and $\beta_{2}$, and once they are known $\theta$ can be found from Figure 6(b) by considering the sum of the internal angles in $A B C$ :

$$
\theta=\frac{\pi-\beta_{1}-\beta_{2}}{2}
$$

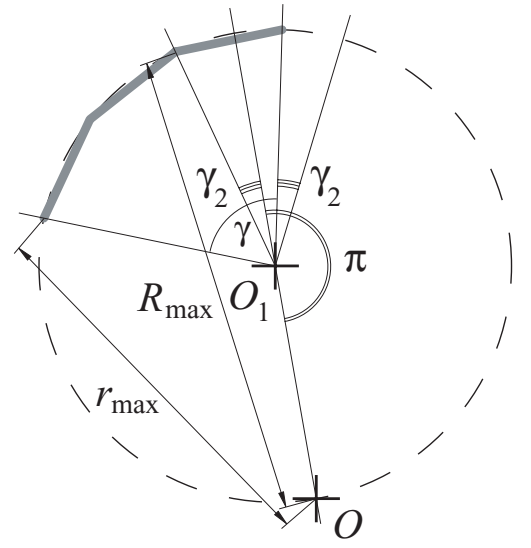

(a)

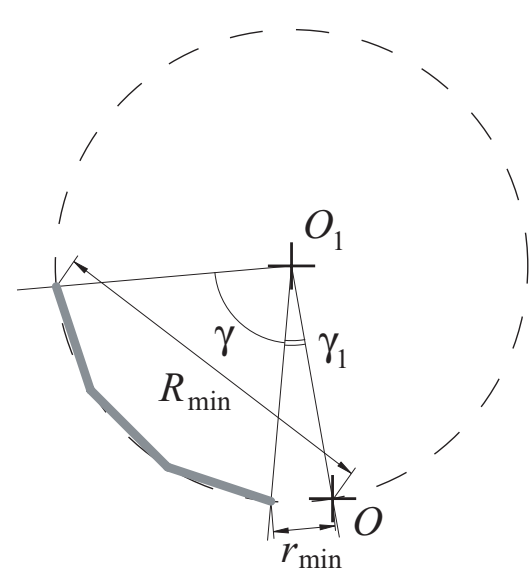

(b)

Figure 5: Definition of maximum and minimum radii and reduction angles 


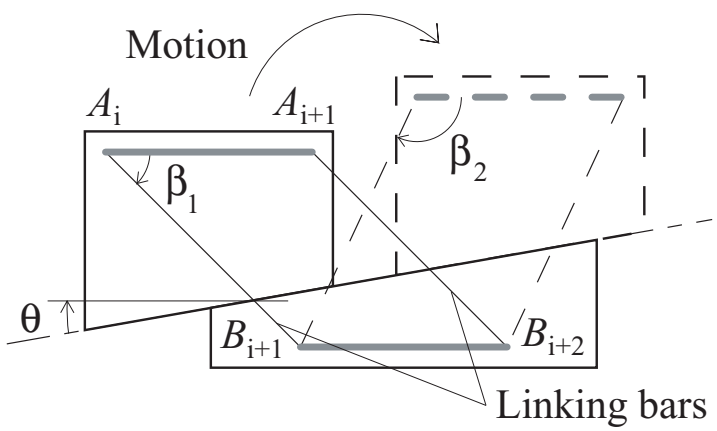

(a)

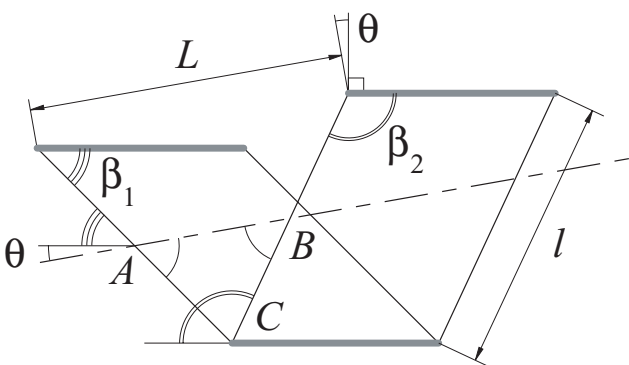

(b)

Figure 6: Motion of simple linkage with two plates

As no length variables are present in Equation 12, the position of the inclination line relative to the linkage does not affect the limits of the motion. Figure 6(b) shows that bar $A_{\mathrm{i}} A_{\mathrm{i}+1}$ translates by a distance $L$ parallel to the inclination line

$$
L=\sqrt{l^{2}+l^{2}-2 l^{2} \cos \left(\beta_{2}-\beta_{1}\right)}=l \sqrt{2-2 \cos \left(\beta_{2}-\beta_{1}\right)}
$$

Next, we consider two neighbouring multi-angulated elements, $A_{1}-A_{5}$ and $B_{1}-B_{5}$, which are part of an expandable bar structure with $k=4$, as shown in Figure 7. Together with bars $A_{1} B_{2}$, $A_{2} B_{3}$, etc., these multi-angulated elements form three interconnected linkages, which are free to move within certain limits.

In the closed configuration, Figure 7(a), the bars $A_{1} A_{2}$ and $B_{1} B_{2}$ form an angle $\alpha$, giving for the first linkage $\beta_{1}{ }^{\prime}=\alpha$. Since the kink angles in the multi-angulated element are equal to $\alpha$, the third linkage has

$$
\beta_{1}^{\prime \prime \prime}=\beta_{1}^{\prime}+(k-2) \alpha=3 \alpha
$$

The limit for the open position is found by noting that bars $A_{3} A_{4}$ and $B_{4} B_{5}$ of the third linkage become collinear and thus $\beta_{2}^{\prime \prime \prime}=\pi$, as shown in Figure 7(b). Therefore,

$$
\beta_{2}^{\prime}=\beta_{2}^{\prime \prime \prime}-(k-2) \alpha=\pi-2 \alpha
$$

A rigid plate is then attached to these interconnected linkages and cut along a single straight line, as before. Now the inclination will be defined with respect to the line $B_{1} B_{2}$, hence the inclination angle is

$$
\theta^{\prime}=\frac{\pi-\beta_{1}^{\prime}-\beta_{2}^{\prime}}{2}+\alpha
$$

Substituting $\beta_{1}^{\prime}=\alpha$ and Equation 15 into Equation 16 we obtain

$$
\theta^{\prime}=(k-1) \frac{\alpha}{2}
$$




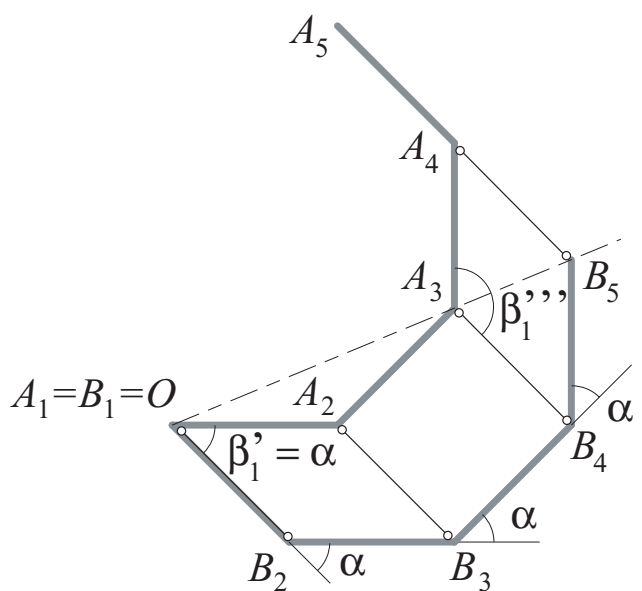

(a)

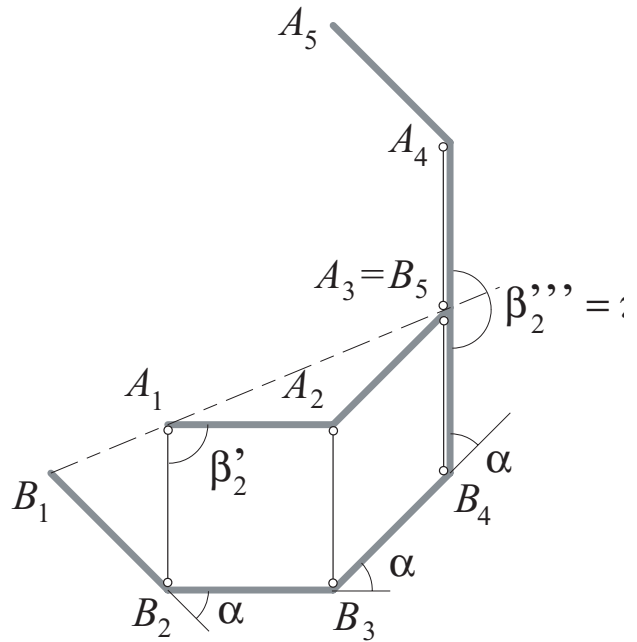

(b)

Figure 7: Two extreme positions of neighbouring angulated elements

Now, turning to the complete bar structure, we consider $n$ inclination lines between the angulated elements. These lines define $n$ identical wedge-shaped covering elements with wedge angle $\alpha$. In the closed configuration these wedges meet at the origin $O$ and form a gapfree surface; in the open configuration they form a gap-free annulus; and they never overlap in any intermediate configurations, as was first observed in Ref. [11].

The reduction angles are included in the definition of the inclination angle by modifying the limits for the linkages:

$$
\begin{aligned}
& \beta_{1}^{*}=\beta_{1}^{\prime}+\gamma_{1} \\
& \beta_{2}^{*}=\beta_{2}^{\prime}-\gamma_{2}
\end{aligned}
$$

Substituting Equations 18 and 19 into Equation 16 the reduced inclination angle is obtained:

$$
\theta^{*}=(k-1) \frac{\alpha}{2}-\frac{\gamma_{1}}{2}+\frac{\gamma_{2}}{2}
$$

From this equation it can be seen that if the two reduction angles are equal, then the solution is a line parallel to that of the original solution in Equation 17.

\section{Shape of Cover Elements}

So far, only straight-edged cover elements have been considered, but in fact non-straight shapes of a periodic type are also possible. Consider the motion shown in Figure 6(a); for the upper and lower cover plates to fit together without any gaps or overlaps in both configurations, the boundary edges must also match in both configurations. Hence, nonstraight features are allowed, provided that they repeat with period $L$, as shown in Figure 8. If the common boundary between the two plates is longer than $L$, then the same features of the lower plate must also be repeated in the upper plate. More generally, plates with common boundaries must be shaped such that all features have a periodic pattern. 


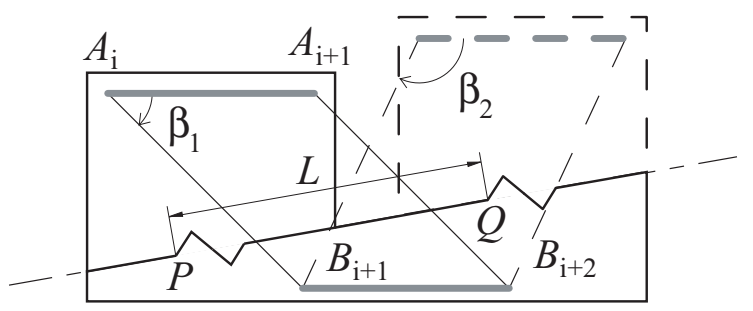

Figure 8: Periodic pattern of non-straight boundary

There are two important restrictions to the above periodicity rule, as obviously a boundary that deviates significantly from the original straight line would inhibit the motion of the linkage. First, when the plates are in contact the initial velocity of any point on the boundary, which is perpendicular to the linking bars, needs to form an angle with the inclination line greater than the slope of the boundary. This is to avoid that the plates jam when the motion is about to begin, Figure 9(a). Second, any deviations of the boundary shape from the original straight line need to lie within a region bounded by two circular arcs that pass through the extreme points $P$ and $Q$ of the repeating length of the boundary; the centres of these circles are defined by the intersections of lines through $P$ and $Q$, and parallel to the linking bars in the open and closed configurations. This is to avoid interference between points on the boundary during the motion of the two plates, Figure 9(b).

The maximum distance, $h$, from the inclination line to the boundary of either plate can be shown to be given by:

$$
h=l\left(1-\sqrt{1-\left(\frac{L}{2 l}\right)^{2}}\right)=l\left(1-\sqrt{\frac{1}{2}+\frac{1}{2} \cos \beta^{*}}\right)
$$

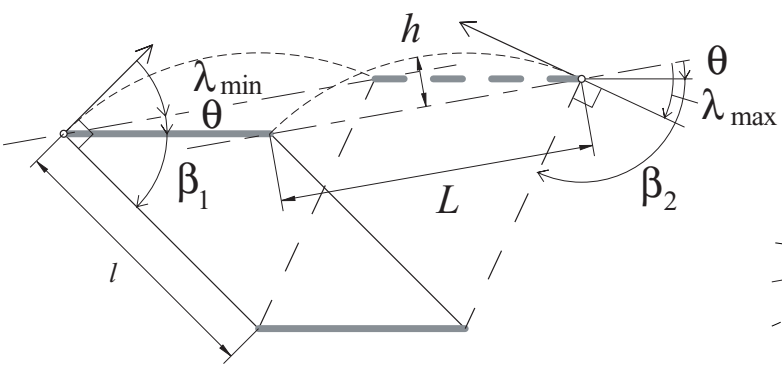

(a)

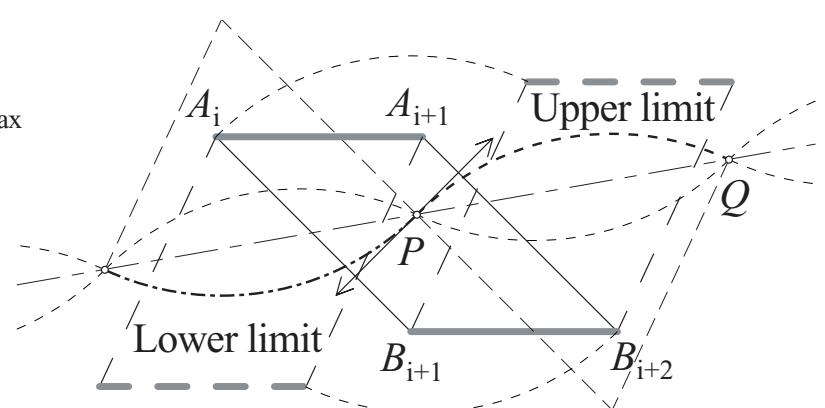

(b)

Figure 9: (a) Direction of velocity vectors in the two extreme positions; (b) region defining possible boundary shapes

\section{DESIGN OF EXPANDABLE STRUCTURES}

Having defined the limits within which the shape of the cover elements can be varied, it is possible to design many different expandable structures. Two different types of structures will be presented. The first is a bar structure covered by a layer of plate elements: as the motion of this structure is controlled by the underlying bar structure, here the cover elements can be fixed to the multi-angulated element in many different ways. The second is purely a plate structure, consisting of two layers of identical plates; this structure is designed such that it is kinematically equivalent to the previous bar structure. 


\section{Bar Structure Covered by Plates}

For cover elements with straight edges the shape of the individual elements is that of a wedge bounded by the inclination lines, which in the closed configuration meet at the origin. Note that in this case, in general, a single cover element will not cover completely the angulated element beneath it.

For cover elements with non-straight edges it is possible to find particular designs that achieve various design aims. For example, it is possible to design a structure that forms a circular opening, in the open configuration. This requires the repeating part of the edge of the cover elements to be a circular arc, since in the open configuration the innermost repeating part forms the edge of the central opening, and the arc radius to be equal to the radius of the opening, $r_{\text {opening. }}$. The smallest possible radius of the boundary $\operatorname{arcs} i s l$, hence the general condition to create a circular opening is

$$
l \leq r_{\text {opening }}
$$

Consider a circle through the tips of the cover elements in the open configuration, which includes the effect of both reduction angles. Hence, effectively adding $\gamma_{1}$ to $\gamma$, the total reduction angle is defined as $\gamma_{\text {tot }}=\gamma_{1}+\gamma_{2}$. The opening radius is then determined from Equation 7

$$
r_{\text {opening }}=2 r^{*} \cos \left[(k-1) \frac{\alpha}{2}+\frac{\gamma_{\text {tot }}}{2}\right]
$$

Substituting Equations 2 and 23 into Equation 22

$$
2 r^{*} \sin \left(\frac{\alpha}{2}\right) \leq 2 r^{*} \cos \left[(k-1) \frac{\alpha}{2}+\frac{\gamma_{\text {tot }}}{2}\right] \Rightarrow \gamma_{\text {tot }} \leq \pi-k \alpha
$$

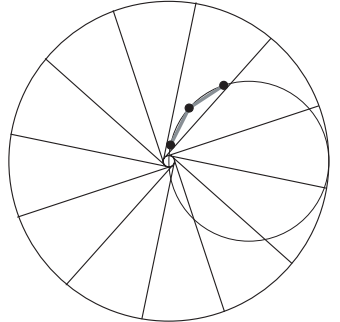

(a)

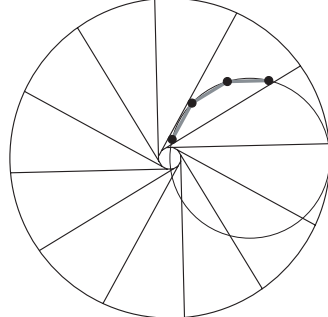

(b)

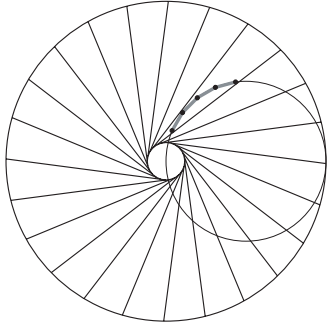

(c)

Figure 10: Wedge shaped plate structures with (a) $k=2$; (b) $k=3$; (c) $k=4$

\section{Plate Structures}

In a plate structure that does not rely on a separate supporting bar structure, it must be possible to fit all joints within the plates, which is often a significant challenge. To date, it has been impossible to construct structures with straight-edged plates and with $k>5$; the solutions that have been found, with $k \leq 4$, are shown in Figure 10. Note that for all these solutions the inclination lines do not meet at the centre, thus leaving a small hole at the centre of the structure in the closed configuration. It is possible to close this opening by modifying the basic wedge shape. 
Using non-straight boundaries it is possible to design structures with a circular opening, as described above, and to minimize the gap, at the centre of the structure, that results from using plates with straight boundaries, see Figure 11.

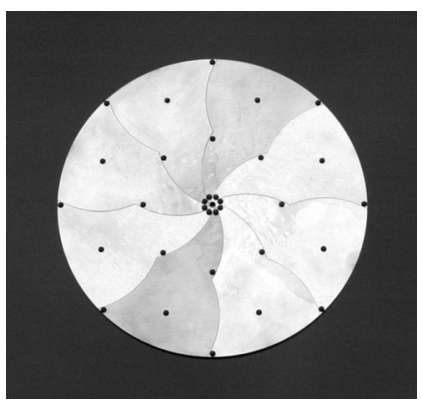

(a)

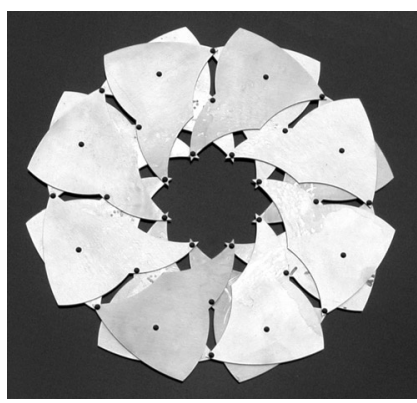

(b)

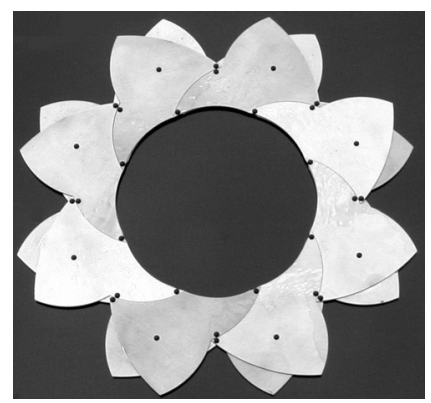

(c)

Figure 11: Model of expandable structure

\section{DISCUSSION AND CONCLUSION}

An analytical approach for finding the shape of hinged plates that can execute large motions has been presented. It has been shown that a large variety of shapes are possible, which are all variants of a simple wedge shape. These expandable plate structures could be used in a wide variety of small and large scale applications where a continuous gap free surface is required that has the ability to execute a large shape change, such as toys, flow and sound control elements and roof structures.

\section{REFERENCES}

1. VERHEYEN H F, A Single Die-Cut Element for Transformable Structures, International Journal of Space Structures, Vol. 8, No. 1 \& 2, 1993, pp 127-134.

2. WOHLHART K, Double-Chain Mechanisms, Proceedings of IUTAM-IASS Symposium on Deployable Structures: Theory and Applications (edited by S Pellegrino and S D Guest), Kluwer Academic Publishers, The Netherlands, 2000, pp. 457-466.

3. YOU Z, A new approach to design of retractable roofs, Proceedings of IUTAM-IASS Symposium on Deployable Structures: Theory and Applications (edited by S Pellegrino and S D Guest), Kluwer Academic Publishers, The Netherlands, 2000, pp. 477-483.

4. ESCRIG F, Arquitectura Transformable, Escuela Técnica Superior de Arquitectura de Sevilla, 1993

5. ESCRIG F and VALCARCEL J P, Geometry of Expandable Space Structures, International Journal of Space Structures, Vol. 8, No. 1 \& 2, 1993, pp 71-84.

6. ZEIGLER T R, Collapsible self-supporting structures and panels and hub therefore, USA Patent no. 4,290,244, 1981.

7. HOBERMAN C, Reversibly expandable doubly-curved truss structure, USA Patent no. 4,942,700, 1990.

8. HOBERMAN C, Radial expansion/retraction truss structures, USA Patent no. 5,024,031, 1991.

9. PELLEGRINO S and YOU Z, Foldable ring structures, Space Structures 4, vol. 4 (edited by G A R Parke and C M Howard), Thomas Telford Publishing, London, 1993, pp. 783-792.

10. YOU Z and PELLEGRINO S, Foldable bar structures, International Journal of Solids and Structures, vol. 34, no. 15, 1997, pp. 1825-1847.

11. KASSABIAN P, YOU Z and PELLEGRINO S, Retractable roof structures, Proceedings Institution of Civil Engineers Structures and Buildings, vol. 134, Feb 1999, pp. 45-56. 